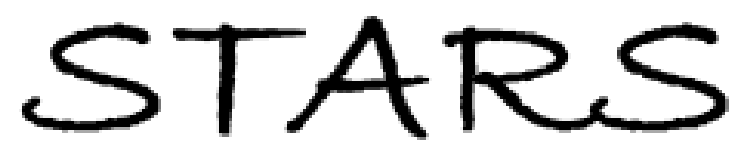

University of Central Florida

STARS

$1-1-2003$

\title{
Angle dependent magnetization reversal of thin film magnetic recording media
}

\author{
Kevin R. Coffey \\ University of Central Florida \\ Thomas Thomson \\ Jan-Ulrich Thiele
}

Find similar works at: https://stars.library.ucf.edu/facultybib2000

University of Central Florida Libraries http://library.ucf.edu

This Article is brought to you for free and open access by the Faculty Bibliography at STARS. It has been accepted for inclusion in Faculty Bibliography 2000s by an authorized administrator of STARS. For more information, please contactSTARS@ucf.edu.

\section{Recommended Citation}

Coffey, Kevin R.; Thomson, Thomas; and Thiele, Jan-Ulrich, "Angle dependent magnetization reversal of thin film magnetic recording media" (2003). Faculty Bibliography 2000s. 3682.

https://stars.library.ucf.edu/facultybib2000/3682

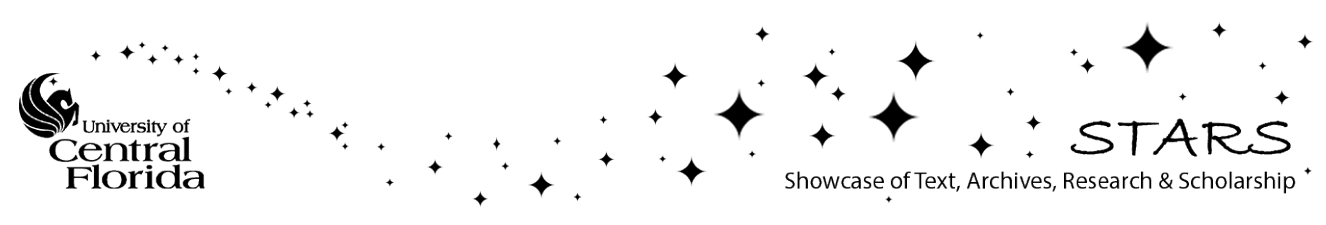




\section{Angle dependent magnetization reversal of thin film magnetic recording media}

Cite as: Journal of Applied Physics 93, 8471 (2003); https://doi.org/10.1063/1.1540167

Published Online: 09 May 2003

Kevin R. Coffey, Thomas Thomson, and Jan-Ulrich Thiele

\section{ARTICLES YOU MAY BE INTERESTED IN}

Angular dependence of the switching field of thin-film longitudinal and perpendicular magnetic recording media

Journal of Applied Physics 92, 4553 (2002); https://doi.org/10.1063/1.1508430

On the modification of the Kondorsky function

Journal of Applied Physics 70, 3184 (1991); https://doi.org/10.1063/1.349301

Angular dependence of the switching field in patterned magnetic elements

Journal of Applied Physics 97, $10 J 705$ (2005); https://doi.org/10.1063/1.1851931

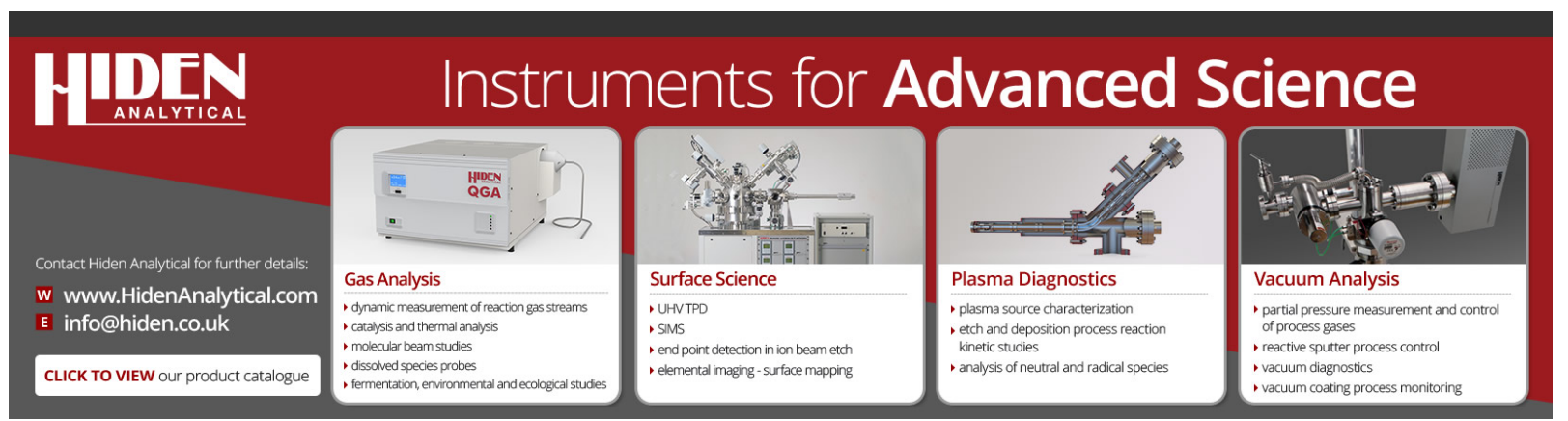




\title{
Angle dependent magnetization reversal of thin film magnetic recording media
}

\author{
Kevin R. Coffeya) \\ University of Central Florida, 4000 Central Florida Boulevard, Orlando, Florida 32816
}

Thomas Thomson and Jan-Ulrich Thiele

IBM Almaden Research Center, 650 Harry Road, San Jose, California 95120

(Presented on 15 November 2002)

\begin{abstract}
The results of angular measurements of the remanent switching fields for granular longitudinal and perpendicular magnetic recording media are presented and compared to idealized models of magnetic switching. Co alloy longitudinal and perpendicular recording media are found primarily to have a Stoner-Wohlfarth switching character at vibrating sample magnetometer time scales. Since the Stoner-Wohlfarth model does not consider the effects of thermal activation, the angular dependence of the time independent switching field $H_{\text {swro }}(\theta)$ was determined from a Sharrock analysis. This approach shows a closer agreement between experimental data and model. For the case of a representative high density longitudinal recording medium, we additionally investigate the switching field distribution. (C) 2003 American Institute of Physics. [DOI: 10.1063/1.1540167]
\end{abstract}

\section{INTRODUCTION}

The optimal writing of media to provide full remanent switching is of interest in designing ultrahigh density magnetic recording systems. While media coercivity is conventionally defined along the easy axis or plane, the fields produced by write heads are vectorial in nature and have components in all three directions. In the case of longitudinal recording with a conventional ring head, this leads to a large angular variation with changes in field magnitude during the writing process. For perpendicular recording with a pole head and soft underlayer, only a small angular variation of the head field is expected. The response of the medium to this angular variation determines the field required for switching and hence affects the placement of the written transition and also, presumably, the sharpness of the written transition. This work will not address the issue of short-time $(\sim$ ns $)$ precessional rotation of magnetization typically described by micromagnetic modeling, ${ }^{1-3}$ but rather provide experimental vibrating sample magnetometry (VSM) data on media switching interpreted using commonly accepted models for the time $e^{4,5}$ and angular dependence. ${ }^{6,7}$ The authors have previously described their experimental method and some results, ${ }^{8}$ and previous workers have also used these models to describe recording media. ${ }^{9,10}$ In this work we present additional data on the switching field distribution for longitudinal media and briefly illustrate the relevance of the angular dependence of media switching to the writing process in magnetic recording.

Figure 1 illustrates the angular dependence of switching for three ideal cases: a collection of Stoner-Wohlfarth particles having a common anisotropy direction, a collection of Stoner-Wohlfarth particles having a two-dimensional (2D)

\footnotetext{
a) Author to whom correspondence should be addressed; electronic mail: krcoffey@mail.ucf.edu
}

isotropic anisotropy distribution, and a (mostly) homogeneous ferromagnetic thin film switching by domain wall motion (Kondorsky switching). The first two models correspond to idealizations of perpendicular and longitudinal recording media, respectively, while the third model describes a strongly exchange coupled thin film. Figure 1 plots the time independent remanent switching field $H_{\text {swro }}(\theta)$ scaled for a convenient comparison of the three cases, as a function of $\theta$, the angle between the applied field and the sample easy axis or plane. In the case of perpendicular media, as well as uniaxial longitudinal media, Stoner-Wohlfarth switching is readily distinguished from Kondorsky switching by the decrease in switching field when the applied field direction is rotated away from the easy axis $\left(\theta=0^{\circ}\right)$. The distinction between Kondorsky and Stoner-Wohlfarth switching for 2D isotropic longitudinal recording media is more subtle. Figure 1 shows a minimum in $H_{\text {swro }}$ at $\theta \cong 40^{\circ}$ and an equality of $H_{\text {swro }}$ at $\theta=0^{\circ}$ and $60^{\circ}$ for $2 \mathrm{D}$ isotropic Stoner-Wohlfarth switching, while for Kondorsky switching there is a monotonic increase in $H_{\text {swro }}$ with $\theta$ and $H_{\text {swro }}$ at $\theta=60^{\circ}$ is twice that of $H_{\text {swro }}$ at $\theta=0^{\circ}$.

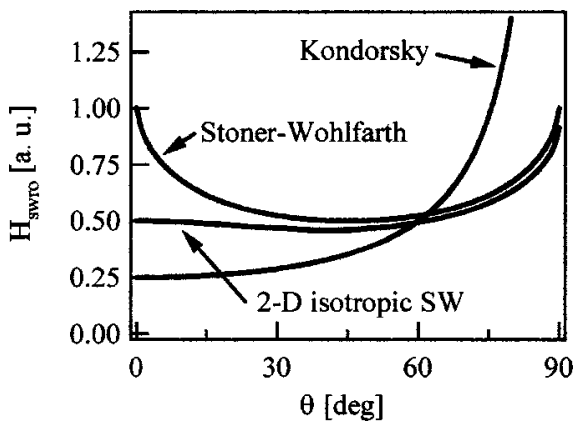

FIG. 1. Relative remanent switching field as a function of angle between the applied field and sample easy axis for Stoner-Wohlfarth, 2D isotropic Stoner-Wohlfarth and domain wall motion (Kondorsky) models of magnetization reversal. 
TABLE I. Remanent coercivity, remanence-thickness product, saturation magnetization, and structural description of the longitudinal and perpendicular media samples.

\begin{tabular}{ccccccc}
\hline \hline Sample & Anisotropy & Alloy & Structure & $H_{C}(\mathrm{Oe})$ & $\begin{array}{c}M_{r} t \\
\left(\mathrm{memu} / \mathrm{cm}^{2}\right)\end{array}$ & $M_{s}\left(\mathrm{emu} / \mathrm{cm}^{3}\right)$ \\
\hline A & longitudinal & CoPtCrB & $(11 \overline{2} 0)$ & 3350 & 0.37 & 330 \\
B & perpendicular & CoPtCr & $(0002)$ & 3750 & 0.86 & 375 \\
\hline \hline
\end{tabular}

\section{SAMPLES}

Experimental data from two representative samples are presented. Sample A is a $\mathrm{CoPtCrB}$ longitudinal recording media on a metal substrate having a $(11 \overline{2} 0)$ crystallographic fiber texture. It is similar to those described by Johnson ${ }^{11}$ and is only approximately $2 \mathrm{D}$ isotropic, having a ratio of circumferential versus radial in-plane remanence of 1.3:1. Sample B is a uniaxial $\mathrm{CoPtCr}$ perpendicular recording media (without a soft underlayer) on a glass substrate similar to those described by Ikeda ${ }^{12}$ where a (0002) crystallographic fiber texture for the recording layer is observed. The remanent coercivity, saturation magnetization, and remanent magnetization-thickness product for the samples are summarized in Table I.

\section{RESULTS AND DISCUSSION}

Figure 2 shows the angle dependent remanent switching in the VSM time frame, $H_{\mathrm{swr}}(\theta)$, and the time independent switching field parameter, $H_{\text {swro }}(\theta)$. The angular range measured is expanded beyond the $0^{\circ}-90^{\circ}$ range to allow observation of symmetry in the presence of experimental errors $\left(\approx 3^{\circ}\right)$ in sample angular position. The time independent data are obtained by extrapolating the remanent coercivity values obtained from a series of remanence curves where the field exposure time is varied between 17 and $109 \mathrm{~s}$, as shown in Fig. 3 for sample A. The remanent coercivity data are modeled using the Sharrock equation ${ }^{4,5,8}$ to yield the time independent switching field which should correspond more

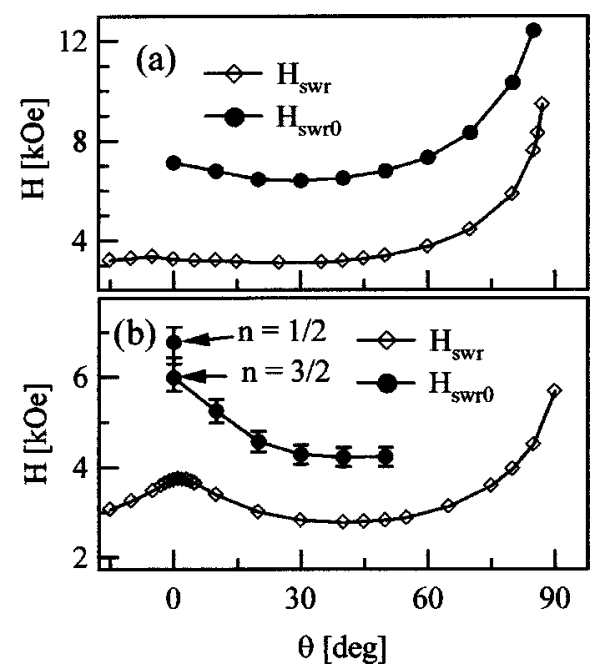

FIG. 2. The remanent switching field and time independent (Sharrock extrapolated) remanent switching field as a function of angle from the easy axis of samples A and B are shown in (a) and (b), respectively. closely to the ideal Stoner-Wohlfarth behavior. The longitudinal medium (sample A) of Fig. 2(a) has a minimum remanent switching field, $H_{\text {swr }}$, near $30^{\circ}$ that is $7.7 \%$ below the in-plane maximum determined from a VSM remanence measurement using a field exposure time of $17 \mathrm{~s}$. This reduction, together with the modest increase in switching field at $\theta$ $=60^{\circ}$, provides a striking resemblance to the $2 \mathrm{D}$ isotropic Stoner-Wohlfarth conceptual model shown in Fig. 1, which exhibits an $8.6 \%$ minimum at $41^{\circ}$. The time independent switching field $H_{\text {swro }}(\theta)$ for this sample has a minimum at $30^{\circ}$ which is $10 \%$ less than the easy axis switching field. This minimum is more pronounced than for the 2D StonerWohlfarth model where a reduction of $8.6 \%$ is expected. We attribute the experimental result to the fact that the medium has an in-plane orientation ratio and that the sample was measured along its preferred, circumferential, in-plane direction.

The angular dependence of $H_{\mathrm{swr}}$ for sample $\mathrm{B}$, shown in Fig. 2(b), also has obvious similarity to the StonerWohlfarth model shown in Fig. 1. Here the minimum in $H_{\text {swro }}(\theta)$ is at $40^{\circ}$ and is $26 \%$ lower than the easy axis value rather than $50 \%$ lower at $45^{\circ}$ as predicted by the StonerWohlfarth model. The methodology used for these measurements ${ }^{8}$ allows comparison of easy axis and hard axis switching and it is clear that the reduction of $H_{\text {swr }}\left(0^{\circ}\right)$ from ideal Stoner-Wohlfarth behavior cannot be fully attributed to a dispersion of easy-axis directions in the sample, ${ }^{10}$ as $H_{\text {swr }}\left(90^{\circ}\right)$ should then be similarly reduced. As with the case of the longitudinal medium, sample A, the time independent switching of the granular perpendicular medium, sample B, shows switching closer to the Stoner-Wohlfarth model. Here the off-axis minimum is either $30 \%$ or $38 \%$ lower than the easy axis value, depending on choice of exponent $(2 / 3$ or $1 / 2$,

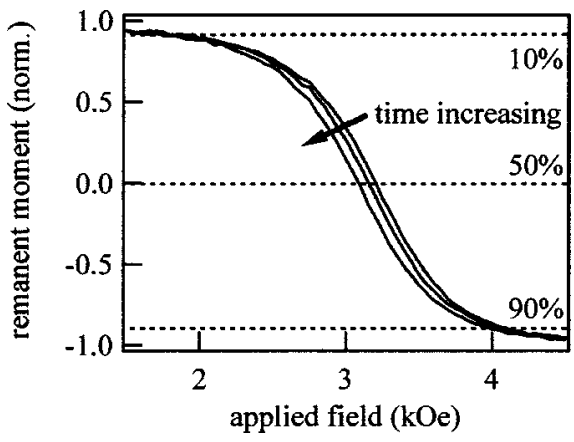

FIG. 3. dc demagnetizing remanence curves as a function of field wait time (17-109 s) for an applied field angle of $\theta=40^{\circ}$. For clarity only three curves are shown. The points at which $10 \%$ and $90 \%$ of the magnetization have switched are also shown, together with the $50 \%$ or coercive point used to define $H_{\mathrm{swr}}(\theta)$. 


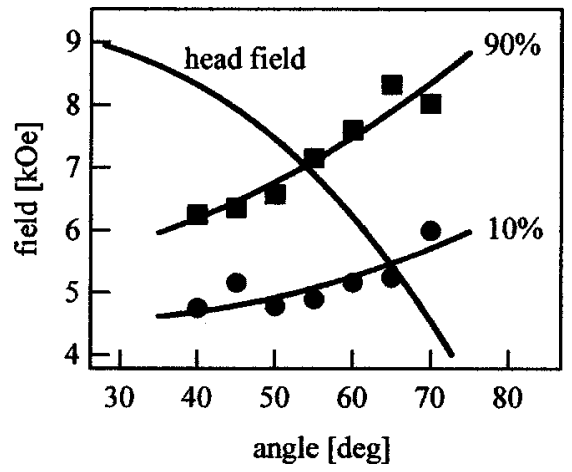

FIG. 4. The time independent (Sharrock extrapolated) remanent switching field distribution of sample A, a longitudinal recording medium, corresponding to sample remanent magnetization being 10\% (squares) and $90 \%$ (circles) switched is shown. Curve segments are added to guide the eye. Also shown is the Karlquist head field calculated for a magnetic spacing of $30 \mathrm{~nm}$, gap of $150 \mathrm{~nm}$, and deep gap field of 15000 Oe.

respectively) used for the easy axis data in the Sharrock equation.

Figure 4 shows data from sample A where this approach has been further extended to look at a time independent switching field distribution. While $H_{\text {swro }}(\theta)$ is derived from the point on remanence curves where the sample is $50 \%$ switched (zero net magnetization), $H_{\text {swro } 10 \%}(\theta)$ and $H_{\text {swro } 90 \%}(\theta)$ can be defined in a similar manner as corresponding to the sample being $10 \%$ and $90 \%$ switched, respectively, ${ }^{9}$ as indicated in Fig. 3 . While this clearly provides information on a sample's time independent switching field distribution, it is an experimentally more difficult measurement than $H_{\text {swro }}(\theta)$, since $d M / d H$ is inherently smaller at the tails of the switching field distribution. The greater noise for these data points is clear in Fig. 4, and short curve segments have been added to the figure to guide the eye. Figure 4 also shows the angular dependence of the head field available to switch the medium according to the Karlquist model of a longitudinal ring head where a magnetic spacing of $30 \mathrm{~nm}$, pole tip gap of $150 \mathrm{~nm}$, and deep gap field of 15000 Oe have been assumed. In this simple model the transition is seen to occur between field angles of approximately $55^{\circ}$ and $65^{\circ}$, which correspond to downtrack positions (from gap center) of 60 and $80 \mathrm{~nm}$, respectively. It is apparent from the figure that the increase in media switching field aids the head field gradient in providing a narrow written transition and that the Stoner-Wohlfarth, rather than Kondorsky switching model more accurately represents the media's response to the head field angle.

\section{CONCLUSION}

The angular dependence of both longitudinal and perpendicular media switching is more consistent with the Stoner-Wohlfarth model than the Kondorsky model. For longitudinal magnetic recording the change in media switching fields across the angular range associated with the written transition width is significant and aids the head field gradient in establishing a narrow transition. A stronger angular dependence of the media switching field near the easy axis is observed for granular perpendicular media and a less dramatic change in head field angle is expected from the pole head/ soft underlayer write head design. In this case it is not clear if the media angular switching dependence aids or hinders the head field gradient in the writing of narrow transitions.

\section{ACKNOWLEDGMENTS}

The authors would like to thank K. Johnson and Y. Ikeda for providing samples used in this work.

${ }^{1}$ Q. Peng and H. N. Bertram, IEEE Trans. Magn. 32, 3566 (1996).

${ }^{2}$ M. Benakli, A. F. Torabi, M. L. Mallary, H. Zhou, and H. N. Bertram, IEEE Trans. Magn. 37, 1564 (2001).

${ }^{3}$ M. Mochizuki, C. Mitsumata, K. Yoshida, A. Nakamura, Y. Maruyama, H. Takano, and H. Aoi, IEEE Trans. Magn. 35, 2520 (1999).

${ }^{4}$ M. P. Sharrock and J. T. McKinney, IEEE Trans. Magn. 17, 3020 (1981).

${ }^{5}$ R. H. Victora, Phys. Rev. Lett. 63, 457 (1989).

${ }^{6}$ E. C. Stoner and E. P. Wohlfarth, Trans. R. Soc. A240, 599 (1948).

${ }^{7}$ E. Kondorsky, J. Phys. (Moscow) II, 161 (1940).

${ }^{8}$ K. R. Coffey, T. Thomson, and J.-U. Thiele, J. Appl. Phys. 92, 4553 (2002).

${ }^{9}$ B. E. Higgins, A. F. Tobari, and M. L. Mallary, IEEE Trans. Magn. 37, 1528 (2001).

${ }^{10}$ T. Shimatsu, H. Uwazumi, Y. Saki, A. Otsuki, I. Watanabe, and H. Muraoka, IEEE Trans. Magn. 37, 1567 (2001).

${ }^{11}$ K. E. Johnson, J. Appl. Phys. 87, 5365 (2000).

${ }^{12}$ Y. Ikeda, Y. Sonobe, G. Zeltzer, B. K. Yen, K. Takano, H. Do, E. E. Fullerton, and P. Rice, IEEE Trans. Magn. 37, 1583 (2001). 